\title{
Management of hyperprolactinaemia in gynaecological practice in blackpool, united kingdom, a study of earlier experience, with a review of relevant literature
}

\begin{abstract}
Aim and objectives: The primary aims of this study were to find out the number of patients (prevalence and incidence) who presented for the first time during the three year period of 2000 to 2002, under review, with hyperprolactinaemia; to assess if their management conformed to any evidence based plan or defined local protocol and whether their management influenced outcome following management. The secondary aim was to formulate a framework template for a protocol on management of patients with hyperprolactinaemia. Such a framework would be used for future audits or reviews on the same subject matter, since this was the first 'audit' or review of its kind in the Unit.
\end{abstract}

Methods: This is a retrospective study of 48 female patients, aged 17-52 years, treated between 2000-2002, who presented with infertility and were found to have hyperprolactinaemia (527-3000 iu/l) at the Blackpool Victoria Hospital, Blackpool, Lancashire, UK.

The duration of hyperprolactinaemia in these patients ranged from 5 months to 36 months with a mean duration of 9 months. The case notes of these women were systematically identified by electronic data and the care they received systematically studied.

5 patients were reviewed first, the rest were reviewed. All case notes were then reviewed three times to ensure consistency in the information retrieved and to minimise errors and omissions. A case report form for each patient was articulated, outlining the relevant demographic data and other information on each patient, including symptoms, investigation, treatment and outcomes of treatment.

Results and conclusion: 48 new female patients presented with raised prolactin levels in 2000-2002 with a mean incidence of about. 0016/10000(+/SD) of the population per year. In 2000, there were 10 cases $(20.83 \%), 18$ cases in $2001(37.50 \%)$ and in 2002 , there were 20 cases $(41.67 \%)$. The incidence had increased and doubled from 2000 to 2002. The prevalence (not part of the table) calculated by including 3 female patients who presented earlier than 2000 but were reviewed as follow-up cases and also managed during the 2000-2002 period, in a population of 329,000 , gives an average estimate of .00 17/10000 $(+/-\mathrm{SD})$ of the population per year. In 6 patients $(12.5 \%)$, macroprolactin was significantly detected (cf $15-26 \%$ of "standard") out of 20 patients (41.67\%), for which fractionation was performed. The incidence of macroprolactinaemia was therefore comparable to the author's cited "standards" in this paper which is $15-26 \%$.

Abbreviations: HGH, Human Growth Hormone; GnRH Gonadotrophin Release Hormone; TSH, Thyroid Stimulating Hormone; IgG, Immunoglobulin G; TRH, Thyrotrophin Release Hormone; MEN, Multiple Endocrine Neoplasia; CT, Computer Tomography; MRI, Magnetic Resonance Imaging; BMD, Bone Mineral Density; BMI, Body Mass Index; IVT-ET, In Vitro Fertilization and Embryonic Transfer; CSF, Cerebrospinal Fluid

\section{Introduction}

Hyperprolactinaemia is defined as a clinical condition with elevated serum prolactin above the reference level established by a given laboratory. It may be asymptomatic as in physiological
Volume 4 Issue 4 - 2016

Anthony Emeka Madu

Department of Obstetrics and Gynaecology, Blackpool Victoria Hospital, UK

Correspondence: Anthony Emeka Madu, Department of Obstetrics and Gynaecology, Blackpool Victoria Hospital, Blackpool, Lancashire, UK, Tel 4479316263I5, Email emymadu@yahoo.co.uk

Received: November 17, 2015 | Published: April 05, 2016

There were inconsistencies and different approaches among various gynaecology teams in the management of these patients and the author believed the management of some patients fell short of the stated author's cited and referenced "standards".

It is the author's opinion that a multidisciplinary approach offers the best outcome for established pathological cases. The multidisciplinary team in the author's view should include Gynaecologist, Obstetrician if the woman is pregnant; Physician, Ophthalmologist if there is ocular involvement, with or without visual impairment; surgeon if a surgical cause was identified; and a psychiatrist, if psychotropic medication (s) was implicated as the cause of hyperprolactinaemia.

Aspects of management of these patients that needed improvement included; history taking, investigation, treatment and follow-up. The author had articulated evidence-based scientific approach for the management of these patients and had additionally included the clinical significance of raised serum macroprolactin. Thus the provisional protocol formulated for the first time was based on available evidence at the time. This protocol, if approved may be updated as new scientific evidence become available.

Keywords: Hyperprolactinaemia, Gynaecological Practice, Blackpool Victoria Hospital Maternity

causes, or symptomatic, presenting with headaches, pressure effects, galactorrhea, menstrual disturbance, infertility, breast tenderness, decreased libido and so on, as in pathological causes. Management is mainly tailored to the cause. However, there are clear indications for management of symptoms and complications. The reference range is largely dependent on the method used by a given laboratory. The reference range for women in Blackpool Victoria Hospital at the time was $83-527 \mathrm{miu} / \mathrm{L}$.

Prolactin is a protein biochemically similar to the human growth hormone $(\mathrm{HGH})$ with a molecular weight of 24,000 . It is secreted by the anterior pituitary lactotrophs and the latter increase in size during pregnancy. Its half-life is $5-10$ minutes. A glycosylated 
form is secreted by the luteal phase endometrium and the decidua of pregnancy. Prolactin is found in three forms in serum according to its molecular weight namely,

a. Monomeric or little prolactin, $23 \mathrm{kDa}$, accounting for $85-100 \%$ of the circulating prolactin, is the physiologically active form.

b. Big prolactin, $50 \mathrm{kDa}, 10-15 \%$.

c. Big, big, prolactin or macroprolactin, produced in small amounts. with a prevalence of $15-26 \%$ in hyperprolactinaemic patients. ${ }^{1-4}$

The dimeric, tetrameric and polymeric forms of prolactin can cause pseudo-hyperprolactinaemia. High levels of prolactin inhibit pulsatile gonadotrophin release hormone $(\mathrm{GnRH})$ secretions by the hypothalamus and oestrogen secreted by the ovary. Prolactin secretion is inhibited by hypothalamic factors especially dopamine, and dopamine antagonists potentiate prolactin release while serotonin and thyroid stimulating hormone (TSH) stimulate prolactin release. Oestogen increase synthesis of prolactin and therefore high doses oestrogen cause elevation of serum prolactin. Though about $30 \%$ of population with secondary amenorrhea have raised serum prolactin but a minority of these have galactorrhea. ${ }^{5}$

Macroprolactin, found in a small number of people, is prolactin complexed with other substances in the blood, most commonly immunoglobulin $\mathrm{G}(\mathrm{IgG})$, protein $\mathrm{A}$, protein $\mathrm{G}$ or antihuman $\mathrm{IgG}$ Some macroprolactin molecules are also glycosylated. It is a cause of moderately elevated prolactin, and patients who have raised serum levels of macroprolactin do not present with classical symptoms of hyperprolactinaemia. They are also less likely to respond to dopamine agonists. The clinical importance of this is that it can cause diagnostic confusion and may lead to unnecessary investigation and treatment. Macroprolactin is also important as some laboratories may detect it as prolactin and this can lead to falsely elevated prolactin result. Tests based on high molecular mass are more successful in detecting macroprolactin than tests based on specific component, like IgG.

The current understanding is that macroprolactin is formed in circulation when prolactin complexes with $\operatorname{IgG}$ and other substances. Macroprolactin is bioactive in vitro but minimally active in vivo. For patients with such condition, treatment with dopamine antagonists (most antipsychotics are dopamine antagonists) for conditions such as schizophrenia, bipolar disorder, and stimulant psychosis cause initial normal acute rise in microprolactin and a later rise in macroprolactin. However, dopamine agonists suppressed microprolactin, with a later falls in macroprolactin.

The responses to both agonist and antagonist actions are likely to be confusing if only total prolactin is measured. Also if we consider that long term response to dopamine agonist is to lower total prolactin levels, more confusion can arise if macroprolactin is not identified and this may reinforce a false notion that treatment had been helpful. This make it imperative for laboratories to determine whether macroprolactin is present and whether the latter is a cause of hyperprolactinaemia as the degree to which macroprolactin contributes to total prolactin varies with individuals and with different assays. ${ }^{6}$ Certain chemicals such as polyethylene glycol can be added to remove macroprolactin from suspicious samples. These samples can then be re-analysed to check if the prolactin levels are still elevated.

Methods for detection of macroprolactin are,

a. Gel filtration chromatography, a classical immunoassay method and gold standard but is time consuming and expensive. b. Measurement of the recovery of prolactin after precipitation with poly ethylene glycol is a fairly crude but widely employed. It precipitates high molecular masses but interferes with some assays.

c. Ultrafiltration, proposed by Prazeres et al. ${ }^{6}$ to overcome problems with using the polyethylene glycol method.

\section{Causes of hyperprolactinaemia}

A. The following can case elevation of serum prolactin, Increases in secretion or release of prolactin, for instance: in pregnancy, during lactation, serotonin and thyrotrophin release hormone (TRH). Antagonism of dopamine, for instance: phenothiazines or most antipsychotics. Reduced clearance of prolactin may occur in chronic renal failure. Depletion of dopamine receptors, for instance: by reserpine and methyldopa can cause increase in prolactin. Blockage of dopamine receptors, for example: sulpiride, haloperidol and metoclopramide. Other mechanisms of hyperprolactinaemia exist aside from those highlighted above and include those highlighted below:

B. Physiological, pharmacological and pathological causes, Physiological causes include sexual intercourse, sleep, vaginal examination, stress, physical examination, breastfeeding, lactation and breast examination. Pharmacological causes include: antipsychotics (neuroleptics)/ antidepressants for instance: phenothiazines, haloperidol, monoamine oxidase inhibitors, selective serotonin re-uptake inhibitors and tricyclic antidepressants (rare cause), opiates, antihypertensives for instance: methyldopa, reserpine and verapamil. Other medications that cause raised prolactin levels are: metoclopramide, domperidone, oestrogen, cocaine, fluoxetine, sulpiride trimethoprim, histamine (H2 receptor) antagonists, bezafibrate, omeprazole.

C. Pathological causes are: Hypothalamic causes include tumours like meningioma, craniopharyngioma, granuloma and dysgerminoma. Non-tumour lesions of hypothalamus include: infections, sarcoidosis and vascular lesions. Pituitory tumours include: prolactinoma in $10 \%$ of cases, acromegaly and nonsecreting adenoma. Other pituitory lesions include: empty sella syndrome, pituitary stalk damage and lyphocystic hypohysis.

D. Other pathological causes are, hypothyroidism, chronic renal failure, hypoadrenalism, cirrhosis of the liver, multiple endocrine neoplasia (MEN), pseudocysis, ectopic prolactin production, polycystic ovarian syndrome (PCOS), Cushing's syndrome and neurogenic causes like spinal cord lesions, chest wall lesions and epilepsy.

About $10 \%$ of the population have asymptomatic prolatinoma, making it imperative to exclude this in a patient with hyperprolatcinaemia. ${ }^{5}$ Prolactomata larger than $10 \mathrm{~mm}$ in diameter are called macroprolactoma and are less frequent in clinical practice. Tumours less than $10 \mathrm{~mm}$ in diameter are microprolactoma: are more frequent in clinical practice and more closely associated with raised prolactin levels.

PCOS is associated with raised prolactin. Amenorrheic women with hyperprolactinaemia have symptoms of oestogen deficiency ${ }^{7}$ but PCOS patients with hyperprolactinaemia have adequate oestrogenization, polycystic ovaries on ultrasound scan and withdrawal uterine bleeding following a progesterone challenge test. Normalisation of TSH is of relevance in the treatment of 
hyperprolactinaemia in hyperprolactinaemic patients with elevated $\mathrm{TSH}^{8}$

Chronic renal failure accounts for a significant number of cases of hyperprolactinaemia. ${ }^{9}$ Many drugs cause a rise in prolactin levels. ${ }^{10}$ Antipsychotic drugs exert their therapeutic effect through antidoperminergic action on the mesolimbic system but their effect in causing hyperprolactinaemia is probably mediated through blockage of dopamine (D2) receptors in the pituitary lactotrophs. ${ }^{11}$ The author believes that as science advances, more drug related causes may became apparent to the scientific community.

\section{Effects of hyperprolactinaemia}

Hyperprolactinaemia may occur without symptoms but symptoms are stated below,

A. Hypogonadism or oestogen deficiency in women and men, Women may have menstrual disturbance which includes amenorrhoea and irregular periods. The commonest pituitory cause of amenorrhea is hyperprolactinaemia. Other presentations are: infertility through anovulation, apathy, vaginal dryness, weight gain and decreased libido. In men, there may be impotence, reduced facial hair, breast enlargement and osteoporosis.

B. Galactorrhea may occur in 33\% of patients but may also be present in patients with normal prolactin levels. Thus its presence does not correlate well with prolactin levels or with the presence of a tumour. ${ }^{12}$

C. Mass effect: this is not due to hyperprolactinaemia per se but to pressure effects of prolactnoma which are also associated with hyperprolactinaemia. These effects include headache, giddiness and visual disturbance through compression of optic nerve if the tumour grows above the pituitary fossa. It can also cause pressure effects if the tumour grows laterally or inferiorly into the cavernous sinus. Visual field defects occur in $5 \%{ }^{13}$ of patients and this is usually bi-temporal hemianopia of the superior quadrants. There may also be palsy of third, fourth and six cranial nerves.

\section{Long term consequences of untreated hyperprolactinaemia}

A. There is low incidence of expansion of prolactinoma but pregnancy has been reported to be associated with tumour expansion, symptomatic or asymptomatic, due to oestrogen stimulation..$^{14}$ It had been reported that tumour regression can occur in pregnancy in relation to vascular changes during and after pregnancy. ${ }^{15}$

B. Progressive reduction in bone mineral density (BMD) is a major long term complication. Trabecular bone is reduced by $10-26 \%$ with a mean of $20 \%$ while cortical bone is reduced by $2.5-11 \%$ with a mean of $6 \%{ }^{16-20}$

The finding of lower BMD in women who have raised prolactin with abnormal menstrual function implicates oestrogen deficiency as a cause of loss of BMD. However, some women who have higher androgen levels have some protection, ${ }^{18,21}$ and in some cases, normalization of prolactin levels leads to increased BMD. According to Klibanski A, et al. ${ }^{15}$ if the degree of hyperprolactinemia was comparable in the hyperprolactinemic women with and without menstrual periods, the mean BMD in hyperprolactinemic amenorrheic women was significantly lower than that in normal women while the mean BMD in the eumenorrheic hyperprolactinemic women though similar to that in the normal women, was significantly greater than that in the hyperprolactinemic amenorrheic women. The mean serum estradiol level in the hyperprolactinemic amenorrheic women was significantly lower than that in the hyperprolactinemic eumenorrheic women measured during the follicular phase of their cycles and was comparable to that in the women with hypothalamic amenorrhea.

\section{Aim and objectives}

The primary aims were to find out the number of patients who presented for the first time during the three year period under review, and to assess if their management conforms to any evidence based practise or to a well-defined and versed local protocol and whether management influenced outcome following management. The secondary aim was to formulate a framework template for a protocol on management of patients with hyperprolactinaemia. Such a framework may be used for future re-audit or review since this was the first audit/review of its kind in the Unit. It may also be regularly updated as new scientific evidence emerge.

\section{Materials and methods}

This is a retrospective study of 48 patients treated at the Blackpool Victoria Hospital for hyperprolactinaemia, from 2000 to 2002.

Patient selection was carefully performed on the basis of elevated prolactin levels from the patient data base on female patients who attended the Department of Gynaecology for fertility and other Gynaecological problem.

There were three other patients who were being followed-up during the time of study. These three patients had presented for the first time before the year 2000 and thus make up the prevalence calculation, though, they were not included in the main study and thus did not form part of the calculation for incidence.

Case report form stated below as a Supplementary Material A, was articulated and then prepared for each patient, outlining the relevant demographic data and other information on each patient, including symptoms, investigations, treatments and outcomes of treatment.

A systematic review of case notes was performed. 5 patients were reviewed first, the rest were then reviewed shortly afterwards. All the case notes were reviewed three times to ensure consistency in the information retrieved and also to minimise errors and omissions.

The results were then analysed and appraised for consistency and accuracy. There was close co-operation and consultation with the clinical biochemistry team, audit department staff, nurses, midwives and library staff and various stages of evolution of the study.

At various stages of evolution of this study, the author met the RCOG Tutor/Consultant Obstetrician and Gynaecologist, the Consultant Biochemist/Clinical Scientist and the Consultant Physician/Endocrinologist to articulate the approach to the study, review and appraise results and any other relevant issues arising from the study. The Blackpool population statistical information was received with by contacting the relevant government body for the statistical information.

Recent research works on the relevant and related topics were obtained through extensive literature search and then studied before analysis of the results. This was essential to blend the study with current information on the subject matter. The Library and Clinical Audit department staff assisted in data presentation at the Clinical Audit meeting. 


\section{Audit standards}

There were no available or known operational guidelines in the management of hyperprolactinaemia at the time of the study (2003 to 2004). Therefore, what formed the "standard" were research works, textbooks and articles from scholars or researchers in the field. Taking good history and performing physical examination including weight, height and BMI. ${ }^{1}$ are important.

These are essential in diagnosis and management as well as exclusion of pregnancy. The latter is essential because pregnancy can lead to elevation of prolactin. It can also be a contraindication to management of elevated prolactin levels or can influence the course of its management.

Systemic review of body systems namely: gastrointestinal, endocrine (thyroid, adrenal, acromegaly or MEN, etc), because a dysfunction in any of these systems may case hyperprolactinaemia. Central Nervous system assessment: as $5 \%$ of patients have headache/ pressure symptoms ${ }^{22}$ Urology and Renal Systems as disorders of these systems can lead to elevation of prolactin levels.

\section{Medication received}

\section{Investigations}

Biochemical: repeat prolactin and measurement of macroprolactin $(15-26 \%$ in the "standard" referenced). Pregnancy test should be performed to exclude an on-going pregnancy. Thyroid function test: as $40 \%$ have thyroid hypofunction ${ }^{3}$ Renal function test: as up to $70 \%$ of patients may have chronic renal failure which is associated with decreased renal clearance of prolactin..$^{23}$ Liver function test: as $5-20 \%$ of patients have alcoholic or non-alcoholic liver cirrhosis. ${ }^{3}$

\section{Radiology examination}

BMD can be performed as $15 \%$ of patients with hyperprolactinaemia have reduces bone density. Lateral skull $\mathrm{x}$-ray may be performed to check for mass intracranial lesions. Computer Tomography/Magnetic Resonance Imaging (CT/MRI) scan should be performed in cases of mild or moderate elevation of prolactin if no other cause was found. X-ray coned view of the sella turcica can be performed if CT/ MRI is not available. Indications for visual field examination are: macroadenoma on CT/MRI, sella turcica enlargement on coned view and moderate elevation of prolactin.

\section{Treatment}

Treatment is targeted to the cause, namely: liver disease, renal disease, endocrine disease, etc. In some cases, the treatment needed is simple observation. Treatment can be categorised with respect to whether pregnancy is desired or not:

I. If pregnancy is not desired, the following had been suggested, Treat with dopamine agonist (bromocriptine). Change to newer agonists like cabergoline and quinagolide) can be done if there is an unacceptable side effect, intolerance or resistance. The dose of medication can be adjusted till ovulation returns. Prolactin levels can be measured every $2-3$ months till ovulation returns, then annually or if anovulation returns.

II. Repeat CT/MRI scans can be performed in the following timeline, For microadenoma: after 1 year, then thereafter, if prolactin levels increase. For macroadenoma: : perform test after 2-3months has passed. When shrinkage is confirmed, repeat test after 1 year, then thereafter if prolactin levels increase. This also include clinical follow-up with prolactin assay every $2-3$ months till prolactin levels fall. Withdraw dopamine agonist after 2-3 years, if there is continuous regular menstruation.

III. If pregnancy desired, the following had been suggested, Before pregnancy: investigation of hyperprolactinaemia includes clinical and radiological evaluation to assess whether there is any tumour. If there is a microadenoma, there is no limitation or contraindications to treatment. For a large adenoma, it is vital for the woman to have evidence of shrinkage before conception. During pregnancy, the advice is to stop treatment with dopamine agonist: perform regular assessments to monitor tumour symptoms like headache and visual disturbance during pregnancy, and then follow-up the patient with routine visual field assessments and other relevant tests. After pregnancy, breast feeding is not contraindicated. However, measurement of prolactin after weaning the child is advised. Treatment of elevated prolactin level is necessary if it occurs in association with anovulation.

\section{Results and analysis of findings}

In 2000 , there were 10 cases $(20.83 \%), 18$ cases in $2001,37.50 \%$ and in 2002, 20 cases, $41.67 \%$. There mean incidence of about $.0016 / 10000(+/-S D)$ of the population per year. The incidence increased and doubled in 2002. The prevalence (not part of the table) calculated by including 3 female patients who presented earlier than 2000 but were reviewed during the 2000-2002 period, in a population of 329,000 , gives an average estimate of $.0017 / 10000$ (+/-SD) of the population per year.

Tables 1-10 below (Supplementary Material B), outlines the salient findings of this study. However, there were other subtle interesting findings worth noting and they are stated below as Supplementary Material C.

Table 1 shows the prolactin levels in the patients with a mean of $837.45 \mathrm{iu} / 1(+/ 28.98)$ and a range of 536-2078 iu/l. Most of the patients have prolactin levels less than $1000 \mathrm{iu} / 1.17$ patients $(35.42 \%)$ have moderate elevation of prolactin between $700 \mathrm{iu} / 1$ and less than $1000 \mathrm{iu} / 1$, while 21 patients $(22.88 \%$ ) have prolactin levels below 700 $\mathrm{iu} / \mathrm{l}$.

Table 2 outline first, the weight of patients with a mean of $70.66 \mathrm{~kg}(+/-8.41)$ and a range of $48-130 \mathrm{~kg}$ for the 11 patients whose weights were documented. One patient was documented as simply overweight. Weight was not stated in 36 patients $(75 \%)$. It also shows the height of 4 patients $(8.33 \%)$ whose heights were recorded and the calculated BMI. The height of 44 patients appeared not to have been documented. The mean height was $166 \mathrm{~cm}(+/-12.88)$.

The ages of patients in table 3 ranged from 17 to 52 years with a mean age of $30.50(+/-5.51)$. Those in age group of 26-30 years account for $27.08 \%$ followed closely by $31-35$ years, $25 \%$ and $20-25$ years, $20.83 \%$

In Table 4, infertility accounts for most frequent complaints established by history $(66.67 \%)$ followed by menstrual disturbance $(52.08 \%)$ and galactorrhoea (14.58\%). Many of these patients presented with more than one symptom at presentation and most of them presenting with infertility also presented with menstrual disturbance and other symptoms.

Table 5 shows some essential investigations. Renal or liver function tests were not performed in 18 patients $(37.50 \%)$ that need these from their history. The same applies to other patients in the table. 
Table 6 outlines the duration of hyperprolactinaemia with a mean duration of 9.40 months (+-3.06). Most patients 29 (60.42\%) have duration of less than 5 months or transient followed by those with a duration of more than 6-12months (14.54\%).

Table 7 shows the causes of hyperprolacinaemia in these patients. The cause was unknown in 33 patients $(68.75 \%)$. Hypothyroidism and antipsychotics were identified as the cause in 3 patients $(6.25 \%)$ each and in 2 patients $(4.16 \%)$, the cause was attributable to stress and surgical operation.

Table 8 outlines the treatment given. Most patients, 32 (68.67\%) needed no treatment and none was rightly offered. No treatment was given to or considered in patients with macroprolactin in their serum.

Table 9 shows the main complaint that made patients present with which is a bit similar to table 4 . Infertility still ranked most frequent at $62.50 \%$, followed by oligomenorrhea which was $12.5 \%$ (cf 30\% in the "standard"), while heavy periods account for $6.25 \%$.

In 28 patients $(58.33 \%)$, the prolactin was not fractioned. This is because PEG method was started in this hospital in August 2001 and the fact that prolactin levels less than $700 \mathrm{iu} / 1$ are not fractioned. In 6 patients $(12.5 \%)$, macroprolactin was significantly detected (cf $15-26 \%$ of "standard") out of 20 patients $(41.67 \%)$ for which the fractionation was performed.

In 4 patients $(8.3 \%)$, macroprolactin was not detected at all with raised microprolactin level, while in 14 patients $(29.16 \%)$ : macroprolactin was detected in insignificant quantity.

One patient had both macroprolactin and microprolactin present in her blood. Though some patients had more than one sample during the period under study, it was the earliest sample that was used for this study.

As stated earlier, there were other subtle but interesting findings and outcomes earlier, from the study in the management of these patients and they are stated below as Supplementary Material C.

\section{Analysis and critique of findings/results}

This is the first study of its kind in the Unit. The study found there were inconsistencies in the management of these patients.

\section{History}

Some written documentations were not legible and readable and made the study more difficult and frustrating. Despite been investigated for raised prolactin, there was no direct/tactical questioning of most patients after they had stated their initial symptoms. For instance: asking about headache, giddiness, visual defects and other pressure symptoms, loss of libido, galactorrhea, etc. With direct questioning (review of systems), absence of these symptoms would be revealed and could influence the course of management, in the opinion of the author.

Such accurate history is vital in following the evolution of the clinical picture at subsequent follow-up visits. These questions may have been asked but they were not documented and there was no other way of knowing whether they were asked.

Menstrual problems and infertility where documented: were probably because patients frequently volunteered them or because that was the main reason for presentation.

\section{Investigation performed}

The following findings were noted, BMI is a comparatively more reliable and essential demographic index in gynaecology than weight alone. It was concerning to note that it was in only 11 patients
(22.91\%) that only actual weight was stated and in one patient it was simply stated as overweight. Height was stated only in 4 patients $(8.32 \%)$ and even in the latter the BMI was not stated. There was no proper timing of sample collection. Basal plasma prolactin level is ideally measured following collection of non-stress venepuncture blood sample from 09-16 hours.

No radiological investigation was performed for 9 patients $(18.72 \%)$ with persistently raised prolactin or before starting medical treatment. There was no consistency in what was the pathological range for raised prolactin for which further investigation can be done. Patients who were symptomatic but with prolactin levels slightly less than $1000 \mathrm{iu} / 1$ were not offered radiological investigation. There was no agreed time frame for a repeat prolactin test for raised prolactin level in case it was diagnostic of the patient's symptoms, or for serial monitoring in case the patient was treated and monitored for resolution of her lesion. There was no consistent format for treatment, investigation (such as biochemical and radiological or invasive procedures) and follow-up even if such was based on level iv evidence. There was no renal and liver function tests performed on patients with persistently raised prolactin levels even if after other causes had been excluded.

In 8 patients $(16.32 \%)$, thyroid function test was not done and there was no attempt to exclude adrenal function in all patients as in the "standard".

3 patients $(6.25 \%)$ have their investigations abandoned because they became pregnant while 5 patients $(10.40 \%)$ with persistently raised prolactin were treated without radiological investigation or other investigation to find out what actually caused the raised prolactin levels before treatment was started.

3 patients $(6.25 \%)$ had raised prolactin with asymmetry of the pituitory fossa or deviation of pituitory stalk and other malformation but no defined adenoma on MRI/CT. This was wrongly assumed from gynaecological point of view, that it was probably idiopathic (cf "standard").

BMD was not performed in many cases with long standing raised prolactin despite been additionally on long term treatment for the raise prolactin. 2 patients $(4.17 \%)$ failed in the follow-up of their investigation and treatment and thus the efficacy of such treatment cannot be verified.

In a significant number of cases, stress was taken as the cause of mild to moderate elevation of prolactin on one single test alone especially postoperatively and after anaesthesia. If there were no further problems, then no further investigation was performed aside from a repeat test of no clear and consistent timing or time frame. This method of testing may then show same prolactin level or lower levels of doubtful diagnostic or follow-up significance. Some patients had investigation for lower levels of prolactin if no other cause was found as a cause of infertility. However, there was no evidence that renal, liver, thyroid and adrenal lesions were excluded as a cause of elevated prolactin levels during clinical assessment.

\section{Medical and surgical treatment}

There were inconsistencies in the response to medical treatment in these patients. 2 patients $(4.17 \%)$ with infertility and mildly raised prolactin were given clomiphene citrate without tubal patency test. One patient who presented with infertility had a normal fallopian tube on one side and with the other tube suspected of being abnormal. She had mild elevation of prolactin and low day 21 progesterone, and was referred for IVF-ET. 
The patient later asked why she was not given clomiphene first and was then given clomiphene. Few patients abandoned their treatment because the prolactin level normalised or they became pregnant.

In some patients, the rise in prolactin level recurred after cessation of treatment.

2 patients $(4.17 \%)$ were referred to the consultant physician based on a single episode of moderately raised prolactin level. Some of these elevations of prolactin could have been stress- related and a repeat test may have revealed a different result like a lower level rather than a persistently raised level.

3 patients $(6.25 \%)$ were on bromocrotine but were later given cabergoline because they were intolerant: resistant to the former or had side effect(s) while on former. 3 patients $(6.25 \%)$ were started on cabergoline directly and had good result despite not having initially had CT/MRI. $6(12.50 \%)$ out of 9 patients $(18.75 \%)$ with persistently raised prolactin level were not transferred to the physician for joint management with the gynaecologist.

2 patients $(4.17 \%)$ tolerated bromocriptine with favourable outcome. One patient $(2.08 \%)$ had radiological test prior to clinical presentation to Gynaecology.

No patient had surgical management.

\section{Discussion}

In general, there was no applicable operational protocol at the Blackpool Victoria Hospital's Department of Obstetrics and Gynaecology, at the time of this study. There were no guidelines from the Royal College of Obstetrician and Gynaecologist or National Institute of Clinical Excellence at the time of this retrospective study. Thus to a large extent, based on the technical definition of audit, this study (the first of its kind in this unit), is essentially a review rather than an audit. Based on the cited articles and textbooks on this subject, a fair critique can thus be articulated.

The management of some of these patients fell below the cited "standards" highlighted in this study and listed below as references. In some cases, it was difficult to ascertain whether the management that was given influenced the eventual outcome due to the way the follow-up was managed. Good or tactful history, therefore, needed to be improved upon. Where indicated, relevant tests like liver, renal, thyroid and adrenal function tests or radiological tests appeared not to have been carried out to rule out other potential causes of hyperprolactinaemia. There were some test results misinterpretations which could have affected eventual outcome of management.

There were 2 clear cases the author believed were errors of clinical judgement in management: one had mild elevation of prolactin and was given clomiphene for several months and when she could not conceive, she was then offered a tubal patency test. The author believes the tubal patency test should have been offered first before offering clomiphene citrate, and that depending on the outcome of the tubal patency test, clomiphene citrate may then be offered if clinically indicated.

The other patient also had mild elevation of prolactin: patent fallopian tubes and borderline day 21 progesterone, and was offered IVF-ET. She later called staff to request clomiphene, saying she would prefer to try clomiphene first. It is the author's view that the patient was correct and had a good chance of conceiving on clomiphene which would then avoid the need more traumatic and expensive IVF-ET.

For cases that are resistant to treatment or cases of persistent mild to moderate elevation of prolactin, it is difficult to conclude whether this was due to poor renal function which accounted for a significant number of cases or due to hypofunction of thyroid, liver and adrenal gland, as tests for the hypofunction of these organ systems were not performed. The hypofunction of these organs are associated with raised prolactin levels.

For long standing cases of hyperprolactinaemia, BMD was not done. BMD is important in assessing long term consequences of hyperprolactinaemia or the efficacy of treatment given. The author observed no clear recognition of the importance of chronic or long standing hyperprolactinaemia in the management of these patients.

Though this study was about women with hyperprolactinaemia, more recent literature review showed that the latter is a common cause of infertility both in females and males with earlier presentation in males compared to females. ${ }^{24-27}$ In males, infertility from hyperprolactinaemia is initially reversible. ${ }^{27,28}$ Hyperprolactinaemia is the most commonly caused by prolactinoma, the latter accounting for $45 \%$ of pituitory tumours. ${ }^{26}$ It is also a cause of infertility in $11 \%$ of oligospermic males ${ }^{29}$ and inhibits pulsatile release of gonadotrophins and testosterone, which in turn leads to low sperm count and impaired sperm motility, function and quality. Hyperprolactinaemia had a defined role in spermatogenesis independent of its influences through gonadotrophins as azoospermia or oligospermia can occur at normal levels of gonadotophins if prolactin levels remained high. ${ }^{28,30}$

After reviewing available literature, Singh $\mathrm{P}$ et al. ${ }^{29}$ advocated the inclusion of prolactin in the guidelines for hormonal evaluation of male infertility and were of the sensible opinion that this assessment should be considered before escalation of investigation to the invasive testicular biopsy test. The author shared this view.

There appear to be disagreement by various authorities on the threshold for imaging in the presence of hyperprolactinaemia, for instance: "Hyperprolacinaemia: Biochemical investigation in laboratory medicine at www.pathology.leedsth.nhs website" ${ }^{31}$ states that: "The choice of $800 \mathrm{mU} / \mathrm{L}$ as a threshold for imaging the pituitary is a compromise and will be affected by clinical features: for example: in women with galactorrhoea/oligomenorrhoea, use a sustained value greater than $600 \mathrm{mU} / \mathrm{L}$ whereas in asymptomatic patients a threshold of $1000 \mathrm{mU} / \mathrm{L}$ may be more appropriate". This threshold and the short algorithmic pathway stated on this site appear to be for women rather than men. It also contrasts with that on this author's provisional protocol at the time of the study. However, the author noted the timeline between this study and the publication referred to. It is also unclear to the author if a different threshold for imaging stated on www.pathology.Leedsth.nhs website, would apply to male patients and why.

More recently, some authorities in the UK and authors around the world in that order, have put out various differing guidelines for management of hyperprolactinaemia, some of which are specific to hyperprolactinaemia caused by medication, ${ }^{32,33}$ while some are nonspecific. These appear to be in recognition of the problem of previous lack of inconsistencies in the approach to management of these patients.

\section{Approach to management/recommendations}

Approach to management should in the opinion of the author depends on,

a. Cause of the raised prolactin

b. Severity of symptoms 
c. Desire for fertility

d. Likelihood of complication from tumour expansion such as headaches, giddiness, visual field defects.

e. Long term consequences of untreated hyperprolactinaemia such as bone demineralization

f. Others.

\section{Provisional protocol for management of hyperprolactinaemia in gynaecology}

History, detailed history legibly documented, including drug, psychiatric, family and social, gynaecological and obstetrical, on first contact is essential if a patient gave a history suggestive or found to have raised prolactin levels.

\section{Investigations, indications are as follows}

a. Symptoms and signs of hypogonadism

b. Infertility with or without pressure symptoms

c. Galactorrhea

d. Thyroid hypofunction

e. Endocrine and hepatic dysfunction

A. Test for prolactin should be repeated in 2-3 weeks depending on the severity of symptoms to exclude transient or physiological causes of raised prolactin. Cannulated prolactin (serial estimation: as per cannulated prolactin protocol) is performed after a second prolactin test had indicated a raised prolactin to rule out stress related causes. If prolactin level is still elevated, a further test or action becomes imperative. Repeat levels of monomeric prolactin more than $700 \mathrm{iu} / 1$ merits imaging referral in the opinion of the author. Prolactin is a good tumour marker and its serum level correlate well with tumour size. In the light of recent information at the time of the study, the local laboratory could establish whether macroprolactinaemia in patients with moderately raised prolactin was the actual cause of hyperprolactinaemia. Timing of prolactin measurement and cannulated prolactin measurement should be in the following time frame: 0900-1600.

B. Tests namely FSH, LH, urea and electrolyte, thyroid and liver function tests, etc.

C. Renal function tests are performed if there is suspicion of chronic renal impairment.

D. Lateral skull X-ray, Microadenomas are usually associated with prolactin elevation (1500-4000iu/1) and are unlikely to be associated with changes on $\mathrm{X}$-ray but macroadenomas secreting prolactin of 5000-8000iu/1 are more likely to be associated with radiological changes. These changes include: asymmetrical enlargement of pituitary fossa, double contour to the floor of pituitary fossa, evasion or erosion of the clinoid process. This investigation is increasingly becoming abandoned for more sophisticated methods like CT and MRI.

E. CT/MRI, These are indicated for: Prolactin levels of 1000iu/1 or more without antecedent history of stress. Prolactin levels of 700iu/l or more with symptoms. Prolactin levels of 700iu/l or more after cannulated prolactin with or without symptoms. These radiological tests help rule out pituitary lesion as a cause of hyperprolactinaemia and allow for detailed examination or marking of the extent of tumour, suprasella extension: compression of optic chiasma and invasion of cavernous sinus.
F. BMD, This should be performed upon diagnosis, to have a baseline, especially if there is a pituitary cause for the raised prolactin. The pituitary cause is associated with greater chronicity of the problem. Serial estimation of BMD at 6 monthly intervals to assess the extent or worsening of osteopenia, progress to frank osteoporosis or to assess resolution after treatment has been commenced. Measurements of BMD can be used to some extent to ascertain the efficacy of treatment as BMD tend to recover following normalisation of prolactin levels. The indications for BMD are prolonged ( $>6$ months) elevation of prolactin, persistently raised prolactin and in those patients with pituitary cause of raised prolactin.

G. Referral for joint care with a physician for causes (a), (c)-(f) above is appropriate.

H. Joint care with a psychiatrist for patient on psychotropics who have raised prolactin levels.

I. Other medications causing raised prolactin can be stopped after assessment of the need to continue medication vis a viz the symptoms the patient have and what happens if the patient stops the medication.

J. For miscellaneous causes, the treatment is with respect to the cause and in most cases removal of the offending stimuli leads to resolution of symptoms without need for medication.

$\mathrm{K}$. Timing of referral for joint or team management should ideally be after diagnosis of the cause of hyperprolactinaemia, unless otherwise recommended.

L. Radiological surveillance should be a joint or team management with the relevant department concerned.

\section{Treatment}

Treatment is with respect to the cause of raise prolactin: adopting strategies to improve renal, thyroid and liver function: stopping intake of the offending drug if necessary, removal of stress. For prolactinomas, observation with clinical and biological surveillance is indicated for small tumours and if gonadal function is normal.

a. For patients with mild symptoms: the aim is to detect small group of patients in this group whose tumour is likely to expand.

b. Medical management, This is indicated for: significant elevation of prolactin $(>700 \mathrm{iu} / \mathrm{L})$, and if symptoms persist or continue. The use of dopamine agonist aims to reducing prolactin secretion by stopping mitotic activity in the pituitary lactotrophs. Shrinkage of the latter is most likely in the first 3 months. However, improvement in the pressure symptoms can be evident within 24-48 hours. ${ }^{22}$ After 3 months, shrinkage is slower except in few cases of large macroadenomas where shrinkage may continue for several months. ${ }^{22}$ Medical treatment is indicated as a prelude to transphenoidal surgery.

c. Cabergoline (D2 agonist), It is superior to bromocriptine (D1 agonist) because of the following, It had a longer time course, is taken once or twice weekly: achieves greater tumour shrinkage: has reduced side effects: has better tolerance: leads to improvement in in visual field defect or visual impairment: has better pregnancy rates and leads to normalisation of prolactin and ovulatory cycles. Cabergoline is also indicated for patients who are resistant to bromocriptine or intolerant of other similar medications. Quinagolide is indicated for cases resistant to bromocriptine or cabergoline. Primary resistance is more 
frequent while acquired resistance though less common may reflect tumour de-differentiation. Apparent resistance usually reflects poor compliance.

For hyperprolactinaemia without prolactinoma: use bromocriptin $1-1.25 \mathrm{mg}$ nocte increasing gradually to $7.5 \mathrm{mg}$ daily in divided doses, maximum of $30 \mathrm{mg}$ daily. For hyperprolactinaemia associated with prolactinoma: use bromocriptin $1-1.25 \mathrm{mg}$ nocte increasing gradually to $5 \mathrm{mg} 6$ hourly, maximum $30 \mathrm{mg}$.

If using cabergoline, start 500ug weekly, singly or in divided doses on separate days, increasing dose monthly if necessary in steps of 500ug till maximum therapeutic response. Alternatively, offer $1 \mathrm{mg}$ weekly $(0.25 \mathrm{mg}-2 \mathrm{mg}$ weekly). The dose can be increased or decreased gradually in cases of intolerance Monitor prolactin levels monthly. If using Quinagolide: start 25ug nocte, for 3 days increasing at intervals for 3 days in steps of $25 \mathrm{ug}$. Maintainance dose is 75 $150 \mathrm{ug}$ daily. For doses higher than $300 \mathrm{ug}$, daily increase in steps of $75-150$ ug at intervals of less than 4 weeks is suggested. ${ }^{26}$

\section{Surgical management of prolactinaemia}

Indications include: resistance to medical management, whether primary, acquired or secondary: intolerance of medical management and complications not manageable medically such as cerebrospinal fluid (CSF) rhinorrhoea, pituitary apoplexy with threat to vision, etc. The cure rates for transphenoidal surgery for macroadenoma are less than that for microadenoma.

Preoperative dopamine agonist for macroadenoma is said to worsens outcomes of surgery due to tumour necrosis.

\section{Summary for treatment of prolactinomata}

Macroadenoma...........Medical.........80\% control of symptoms

Surgical......26\% cure........risks of morbidity/mortality.

Surgery........reserved for intolerance to medical treatment or complications like CSF rhinorrhoea/apoplexy.

Microadenoma.......Medical......80-90\% control of symptoms.

Surgical...........60\% risk of hypopituitarism requiring life-long replacement therapy.
Surgery........reserved for poor tolerance or intolerance of medical treatment.

\section{Supplementary material A}

Case Report Form,

Name......... weight age. height. body mass index (BMI)

Symptoms and duration

Headache

Other pressure effects/symptoms

Galactorrhea

Menstrual disturbance

Infertility

Breast Tenderness

Decreases libido

Dyspareunia

Others symptoms and signs....

Obstetric history, parity and other relevant information

Biochemistry

Renal status

Radiology

Prolactin and macroprolactin levels

Bone densitometry

Duration of hyperprolactinaemia

Cause or probable cause of hyperprolactinaemia

Treatment given or offered

Main reason for presentation in hospital

Outcome/effect of treatment

\section{Supplementary material B}

Table I Levels of prolactin

\begin{tabular}{lll}
\hline Prolactin level(iu/L) & Number of patients & $\%$ \\
\hline$<1000$ & 38 & 79.17 \\
$1000-3000$ & 10 & 20.08 \\
$>3000$ & 0 & 0 \\
\hline
\end{tabular}

Table 2 Weight of patients/Height and BMI of patients for 4 patients in which it is possible to measure BMI

\begin{tabular}{lll}
\hline Weight $(\mathrm{kg})$ & Number of patients & $\%$ \\
\hline$<50$ & 2 & 4.17 \\
$50-100$ & 7 & 14.58 \\
$>100$ & 2 & 4.17 \\
unstated & 36 & 75 \\
Weight $(\mathrm{kg})$ in 4 patients & Height $(\mathrm{cm})$ in 4 patients & BMI $(\mathrm{kg} / \mathrm{cm} 2)$ in 4 patients \\
60 & 159 & 24 \\
57 & 161 & 22 \\
64 & 169 & 23 \\
130.8 & 173 & 43 \\
\hline
\end{tabular}


Table 3 Ages of patients

\begin{tabular}{lll}
\hline Age & Number of patients & $\%$ \\
\hline$<20$ & 3 & $6.25 \%$ \\
$20-25$ & 10 & $20.83 \%$ \\
$26-30$ & 13 & 27.08 \\
$31-35$ & 12 & 25 \\
$36-40$ & 6 & 12.5 \\
$4 I-50$ & 3 & 25 \\
$>50$ & 1 & 2.08 \\
\hline
\end{tabular}

Table 4 Presentations consistent with hyperprolactinaemia

\begin{tabular}{llll}
\hline Presentation & Number of patients it is present in Number of patients it is absent in & Not stated \\
\hline Infertility & $32(66.67 \%)$ & $2(4.17 \%)$ & $14(29.16 \%)$ \\
Menstrual Disturbance & $25(52.08)$ & $21(43.75 \%)$ & $2(4.17 \%)$ \\
Galactorrhea & $7(14.58 \%)$ & $11(22.92 \%)$ & $29(60.42 \%)$ \\
Headache & $6(12.50 \%)$, cf 5\% in "standard" & $6(12.50 \%)$ & $36(75 \%)$ \\
Other Pressure Symptom & $3(6.25 \%)$ & $10(20.83 \%)$ & $35(72.92 \%)$ \\
Dyspareunia & $4(8.3 \%)$ & $1(2.08 \%)$ & $42(89.36 \%)$ \\
Breast Tenderness & $1(2.08 \%)$ & 0 & $47(97.92 \%)$ \\
Libido & $1(2.08 \%)$ & 0 & $47(97.92 \%)$ \\
\hline
\end{tabular}

NB, Some of these presentations may be the direct cause, example: hypothyroidism and surgery, or in association, example: PCOS, or the cause of medication used in treating the disease itself, example: bipolar affective disorder and complex partial seizures.

Table 5 Investigations conducted

\begin{tabular}{lllll}
\hline Investigation & Done & Not done & Indicated & Not indicated \\
\hline Renal/Liver Function & $\mathrm{I}(2.08 \%)$ & $47(97.92 \%)$ & $19(39.58 \%)$ & $29(60.42 \%)$ \\
Thyroid Function & $10(20.83 \%)$ & $38(79.17 \%)$ & $19(39.58 \%)$ & $29(60.42 \%)$ \\
Radiology & $9(18.75 \%)$ & $39(81.25 \%)$ & $18(37.50 \%)$ & $30(62.25 \%)$ \\
Bone Mineral Density (BMD) & I (2.08\%) & $47(97.92 \%)$ & $10(20.83 \%)$ & $38(79.19 \%)$ \\
\hline
\end{tabular}

Table 6 Duration of hyperprolactinaemia

\begin{tabular}{lll}
\hline Duration of hyperprolactinaemia & Number of patients & $\%$ \\
\hline$<5$ Months/Transient & 29 & 60.42 \\
$5-6$ Months & 5 & 10.42 \\
$>6$ Months -12 Months & 7 & 14.58 \\
$>12$ Months -24 Months & 3 & 6.25 \\
$>24$ Months-36 Months & 4 & 8.33 \\
\hline
\end{tabular}

Table 7 Causes of hyperprolactinaemia

\begin{tabular}{lll}
\hline Cause of hyperprolactinaemia & Number of patients & $\%$ \\
\hline Unknown/not Stated & 33 & $68.75 \%$ \\
Hypothyroidism & 3 & 6.25 \\
Antipsychotics & 3 & 6.25 \\
Stress/Surgery & 2 & 4.17 \\
PCOS & 2 & 4.17 \\
PCOS/Antipsychotic & 1 & 2.08 \\
Pregnancy & 1 & 2.08 \\
Contraceptive Pill & 1 & 2.08 \\
Pituitary Disease/Lesion & 2 & 4.17 \\
\hline
\end{tabular}

Table 8 Treatment given

\begin{tabular}{lll}
\hline Treatment (Mainly medical) & Number of patients & $\%$ \\
\hline Bromocriptine/Cabergoline/Change of Antipsychotic & $\mathrm{I}$ & 2.08 \\
Reduce Antipsychotic & $\mathrm{I}$ & 2.08 \\
Bromocriptine/Cabergoline & 3 & 6.25 \\
Cabergoline & 3 & 6.25 \\
Rejected Treatment & $\mathrm{I}$ & 2.08 \\
Pill+ Referral to Dietician & $\mathrm{I}$ & 2.08 \\
Clomiphene & 2 & 4.17 \\
Referred to Physician & 4 & 8.33 \\
Not Stated/None & 32 & 66.67 \\
\hline
\end{tabular}

Citation: Madu AE. Management of hyperprolactinaemia in gynaecological practice in blackpool, united kingdom, a study of earlier experience, with a review of relevant literature. Obstet Gynecol Int J. 20 I6;4(4): I08-I I 8. DOI: I0.15406/ogij.20। 6.04.00 I I2 
Table 9 Main presenting complaint

\begin{tabular}{lll}
\hline Main presentation & Number of patients & $\%$ \\
\hline Infertility & 30 & 62.5 \\
Oligomenorrhoea/Amenorrhoea & 6 & $12.50 \mathrm{cf} 30 \%$ in "standard" \\
Heavy Painful Periods & 3 & 6.25 \\
Recurrent Miscarriage & 2 & 4.17 \\
Headache/Visual Problem & $\mathrm{I}$ & 2.08 \\
Galactorrhea & $\mathrm{I}$ & 2.08 \\
Galactorrhea/Dyspareunia & $\mathrm{I}$ & 2.08 \\
Infertility/Hot Flushes & $\mathrm{I}$ & 2.08 \\
Dyspareunia & $\mathrm{I}$ & 2.08 \\
Irregular Painful Periods & $\mathrm{I}$ & 2.08 \\
Headaches/Breast Soreness & $\mathrm{I}$ & 2.08 \\
\hline
\end{tabular}

\section{Supplementary material C}

Other subtle and interesting findings and outcomes from the study in the management of these patients

*Infertility, primary $21(43.75 \%)$, secondary $11(22.92 \%)$.

Decrease in prolactin level/still amenorrheic, 1 patient $(2.08 \%)$.

Pregnant while waiting for In vitro fertilization and embryonic transfer (IVF-ET), 2 patients (4.17\%).

Referred for IVT-ET, 3 patients $(6.25 \%)$.

Decreased prolactin and irregular periods, 6 patients (12.50\%).

Pregnancy after reduction of dose of antipsychotic, 1 patient $(2.08 \%)$

Decreased prolactin, then pregnancy, recurrent hyperprolactinaemia and menopause in that order, 1 patient $(2.08 \%)$.

Pregnancy after 5 months, had regular period, 1 patient (2.08\%).

Partner azoospermic, 1 patient (2.08\%).

Partner oligospermic, 1 patient $(2.08 \%)$.

Partner with Klinefelter syndrome, 1 patient (2.08\%).

Decreased headache, new glasses, 1 patient $(2.08 \%)$.

Decreases prolactin in 3 patients $(6.25 \%)$ on follow-up.

\section{Other presentations found in women with hyperprolactinaemia during this study,}

Bipolar affective disorder, 1 patient (2.08\%).

PCOS/Past history of PCOS, 4 patients $(8.33 \%)$.

Tiredness as main presentation, 1 patient $(2.08 \%)$.

Sleep disorder and hypothyroidism, 1 patient $(2.08 \%)$.

Pelvic pain/lower abdominal pain, 1 patient (4.17\%).

Hypothyroidism/tuberous sclerosis/obesity, 1 patient (2.08\%).

Complex partial seizure, 1 patient (2.08\%).

Surgery/anaesthesia at the time of test 2 patients (4.17\%).

History of craniopharyngioma/hypothyroidism, 1 patient (2.08\%)

Thus total number of patients with hypothyroidism is $3(6.25 \%)$, cf $40 \%$ in "standard."

Bilateral galatorrhea with normal prolactin levels, 1 patient $(2.08 \%)$
Total number of patients with galactorrhea is $8(16.67 \%)$ cf $33 \%$ in "standard."

Total number of patients with decreased prolactin and resumption of regular menstruation, 11 patients $(22.92 \%)$ cf $70 \%$ in Molith ME3 though one of these patients remained amenorrheic.

Failure to comply with follow-up, 3 patient $(6.25 \%)$.

General practitioner follow-up, 1 patient (2.08\%).

Follow-up by physician, 2 patients $(4.17 \%)$.

Follow-up not stated in 18 patients $(37.50 \%)$.

\section{Special thanks to,}

Mr SJ Duthie, RCOG Tutor/ Consultant Obstetrician and Gynaecologist

Dr S Butler, Consultant Biochemist/Clinical Scientist

Dr Abu Ahmed, Consultant physician/Endocrinologist

Midwives in Ward D

Gynaecology Nurses

\section{Acknowledgments}

None.

\section{Conflicts of interest}

None.

\section{References}

1. Whitefield CRD. Dewhurst Textbook of Obstetrics and Gynaecology for postgraduates (5th edn), Oxford Blackwell publishing, United kingdom. 1995;p.37.

2. Olukoga AO, Kane JW. Macrorolactinaemia, validation and application of polyethylene glycol precipitation test and clinical characterization of the condition. Clin Endocrinol. 1999;51(1):119-126.

3. Bjoro T, Morkrid K Wereland R, Turter A, et al. Frequency of Hyperprolactinaemia due to large molecular weight prolactin (150-170 kDa PRL). Scand J Clin Invest. 1999;55(2):139-147.

4. British National Formulary. United kingdom. 2003;45:374.

5. Fahie WMN, Ahlquist JA. Hyperprolactinaemia due to macroprolactin, some progress but still a problem, Clin Endocrinol. 2003;58(6):683-685.

6. Prazeres S, Santos MA, Ferreira HG, et al. A practical method for detection of macroprolactin using ultrafilteration. Clin Endocrinol. 2003;58(6):686-690. 
7. Raber W, Gessl A, Nototny P, et al. Hyperprolactinaemia in hypothyroidism, Clinical significance and Impact of TSH normalization. Clin Endocrinol. 2003;58(2):185-191.

8. Annonymous, Drug related hyperprolactinaemia. Prescrire international. 2003;12(63):18.

9. Wieck A, Haddad P. Hyperprolactinaemia caused by antipsychotic drugs, BMJ. 2002;324(7332):250-252.

10. Jacobs HS, Frank S, Murray MAF, et al. Clinical and endocrinologic features of hyperprolactinaemic amenorrhea. Clin Endocrinol. 1976;5(5):439-454.

11. Edmond DK. Dewhurst Textbook of Obstetrics and Gynaecology for postgraduates. (6th edn), Oxford, Blackwell publishing, United kingdom. 1999;p:54-58.

12. Molitch ME. Pregnancy and the hyperprolactinaemic woman. $N$ Engl J Med. 1985;312(21):1364-1370.

13. Jeffcoat WJ, Pound N, Sturrock NDC, et al. Long term follow-up of patients with hyperprolactinaemia. Clin Endocrinol. 1996;45(3):299303.

14. Klibanski A, Neer RM, Beitins IZ, et al. Decreased Bone Density in hyperprolactinaemic women. $N$ Engl J Med. 1980;303(26):1511-1514.

15. Klibanky A, Greenspan SL. Increase in bone mass after treatment of hyperprolactinaemic amenorrhoea. N Engl J Med. 1986;315(9):542546.

16. Klibanski A, Biller BMK, Rosenthal DI, et al. Effects of prolactin and oestrogen deficiency in amenorrheic bone loss. J Clin Endocrinol Metab. 1988;67(1):124-130.

17. Biller BMK, Baum HBA, Rosenthal DI Saxe VC, et al. Progressive trabecular osteopenia in women with hyperprolactinaemia. $J$ of Clinical endocrinology and metabolism. 1992;75(3):692-697.

18. Webster J, Baillier's. Clinical Endocrinology and Metabolism. 1999;13(3),395-408

19. Bevan JS, Webster J, Burke CW, et al. Dopamine agonist and pituitary tumour shrinkage. Endocr Rev. 1992;13(2):220-240.

20. Fahie-Wilson MN, Soule SG. Macroprolactinaemia, Contribution to hyperprolactinaemia in a District General Hospital and evaluation of a screening test based upon precipitation with polyethylene glycol. Ann Clin Biochem. 1997:34(3):252-258.
21. Wass JAH, Shale SM. Oxford Textbook of Endocrinology and Diabetes (1st edn), United kingdom. 2002;p:1130-1133.

22. Smith TP, Suliman AM, Fahie-WMN, et al. Gross variability in the detection of prolactin in sera containing big big prolactin (macroprolactin) by commercial immunoassays. J Clin Endocrinol Metab. 2002;87(12):5410-5415.

23. Ciccarelli A, Daly AF, Beckers A. The epidemiology of prolactinomas. Pituitary. 2005;8(1):3-6.

24. Buvat J. Hyperprolactinemia and sexual function in men, a short review. Int J Impot Res. 2003;15(5):373-377.

25. Soler FJM, Caravaca MF, Domínguez BC, et al. Correlation of serum prolactin, sperm count and motility.Prevalence of hyperprolactinemia in the infertile male. Arch Esp Urol. 1990;43(8):891-895.

26. Masud S, Mehboob F, Bappi MU. Severe hyperprolactinemia directly depresses the gonadal activity causing infertility. Esculapio J Services Inst Med Sci. 2007;2(4):25-27.

27. Hyperprolacinaemia. Biochemical investigation in laboratory medicine. 2015.

28. Guidance on the TreatmentofAntipsychotic Induced Hyperprolactinaemia in Adults. Version 1. 2014

29. Singh P, Singh M, Cugati G, et al. Hyperprolactinemia, An often missed cause of male infertility. J Hum Reprod Sci. 2011;4(2):102-103.

30. Guidelines for the Management of antipsychotic-induced hyperprolactinaemia. NHS. 2013.

31. Salazar-LOCG, Hernández-BJA, González-BD, et al. Clinical Practice Guideline for the diagnosis and treatment of hyperprolactinaemia. Ginecol Obstet Mex. 2014;82(2):123-142.

32. Halperin RI, Cámara GR, García MM, et al. Clinical Guideline for the diagnosis and treatment of prolactinoma and hyperprolactinaemia. Endocrinol Nutr. 2013;60(6):308-319.

33. Jacobs HS. Management of prolactin secreting tumours. In, stud J (Ed.), Progress in Obstetrics and Gynaecology, Edinburgh, Churchill, Livingstone, Scotland. 1981;1:263-276. 